\title{
A propensity-matched analysis comparing survival after primary minimally invasive esophagectomy followed by adjuvant therapy to neoadjuvant therapy for esophagogastric adenocarcinoma
}

\author{
Haris Zahoor, MD, ${ }^{\mathrm{a}}$ James D. Luketich, MD, ${ }^{\mathrm{b}}$ Ryan M. Levy, MD, ${ }^{\mathrm{b}}$ Omar Awais, DO, \\ Daniel G. Winger, MS, ${ }^{\mathrm{c}}$ Michael K. Gibson, MD, PhD, ${ }^{\mathrm{d}}$ and Katie S. Nason, MD, MPH ${ }^{\mathrm{b}}$
}

\begin{abstract}
Objectives: Prognosis for patients with locally advanced esophagogastric adenocarcinoma (EAC) is poor with surgery alone, and adjuvant therapy after open esophagectomy is frequently not tolerated. After minimally invasive esophagectomy (MIE); however, earlier return to normal function may render patients better able to receive adjuvant therapy. We examined whether primary MIE followed by adjuvant chemotherapy influenced survival compared with propensity-matched patients treated with neoadjuvant therapy.
\end{abstract}

Methods: Patients with stage II or higher EAC treated with MIE $(\mathrm{N}=375)$ were identified. Using 30 pretreatment covariates, propensity for assignment to either neoadjuvant followed by MIE $(\mathrm{n}=183 ; 54 \%)$ or MIE as primary therapy $(\mathrm{n}=156 ; 46 \%)$ was calculated, generating 97 closely matched pairs. Hazard ratios were adjusted for age, sex, body mass index, smoking, comorbidity, and final pathologic stage.

Results: In propensity-matched pairs, adjusted hazard ratio for death did not differ significantly for primary MIE compared with neoadjuvant (hazard ratio, $0.83 ; 95 \%$ confidence interval, $0.60-1.16$ ). Recurrence patterns were similar between groups and $65 \%$ of patients with IIb or greater pathologic stage received adjuvant therapy. Clinical staging was inaccurate in 37 out of 105 patients $(35 \%)$ who underwent primary MIE $(n=18$ upstaged and $n=19$ downstaged).

Conclusions: Primary MIE followed by adjuvant chemotherapy guided by pathologic findings did not negatively influence survival and allowed for accurate staging compared with clinical staging. Our data suggest that primary MIE in patients with resectable EAC may be a reasonable approach, improving stage-based prognostication and potentially minimizing overtreatment in patients with early stage disease through accurate stage assignments. A randomized controlled trial testing this hypothesis is needed. (J Thorac Cardiovasc Surg 2015;149:538-47)

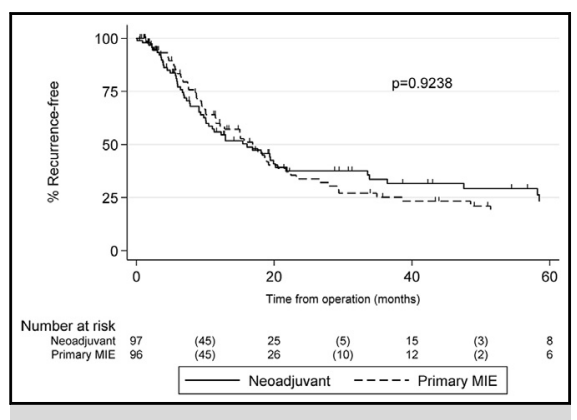

Survival of propensity-matched groups.

\section{Central Message}

Primary minimally invasive esophagectomy produced more accurate staging and did not negatively impact survival compared with clinical staging.

\section{Clinical Relevance}

Primary minimally invasive esophagectomy followed by adjuvant therapy in patients with locally advanced esophagogastric adenocarcinoma may be a reasonable alternative to treating all with neoadjuvant therapy. This approach permits accurate stage assignment and could improve prognostication and minimize the risk of overtreatment in patients with early-stage disease.
From the Departments of Medicine ${ }^{\mathrm{a}}$ and Cardiothoracic Surgery, ${ }^{\mathrm{b}}$ and Clinical and Translational Science Institute, ${ }^{c}$ University of Pittsburgh, Pittsburgh, Pa; and the Department of Medicine, Case Western Reserve University, ${ }^{\mathrm{d}}$ Cleveland, Ohio.

Supported by award Nos. K07CA151613 (to K.S.N.), UL1 RR024153, and UL1TR000005 from the National Cancer Institute. The content is solely the responsibility of the authors and does not necessarily represent the official views of the National Cancer Institute or the National Institutes of Health.

Disclosures: James D. Luketich reports equity ownership in Intuitive Surgical. Omar Awais reports consulting fees from Baxter and lecture fees from Covidien. All other authors have nothing to disclose with regard to commercial support.

Read at the Residents \& Fellows Research Conference and Plenary Session of the 53rd Annual Meeting of the Society for Surgery of the Alimentary Tract, San Diego, California, May 18 and 21, 2012.

Received for publication Feb 14, 2014; revisions received Sept 22, 2014; accepted for publication Oct 6, 2014; available ahead of print Nov 24, 2014.

Address for reprints: Katie S. Nason, MD, MPH, 5200 Centre Ave, Ste 715, Shadyside Medical Building, Pittsburgh, PA 15232 (E-mail: nasonks@upmc.edu).

$0022-5223 / \$ 36.00$

Copyright (c) 2015 by The American Association for Thoracic Surgery

http://dx.doi.org/10.1016/j.jtcvs.2014.10.044
The rationale for trimodality therapy in the management of esophagogastric adenocarcinoma (EAC) is treatment of systemic micrometastasis and tumor downstaging, thus increasing the likelihood of complete resection, local control, and overall survival. ${ }^{1-4}$ Patients who maintain a reasonable performance status after neoadjuvant chemo(radio)therapy undergo esophagectomy with regional lymphadenectomy. In theory, this rationale is sound. In practice, great variability in neoadjuvant regimens exists between centers, and when studied in randomized controlled trials, comparisons between multimodal therapy and surgery alone yield conflicting results. ${ }^{6-11}$ Lack of consistent neoadjuvant regimens is compounded by the limitations of clinical staging, ${ }^{12-17}$ and differences in approach to esophagectomy, extent of lymphadenectomy, and perioperative outcomes 


\section{Abbreviations and Acronyms \\ AJCC $=$ American Joint Committee on Cancer \\ CCI $=$ Charlson Comorbidity Index \\ $\mathrm{CT}=$ computed tomography \\ EAC = esophagogastric adenocarcinoma \\ EUS = endoscopic ultrasound \\ MIE = minimally invasive esophagectomy \\ PET = positron emission tomography}

between centers. ${ }^{18,19}$ Finally, lack of proven adjuvant therapies and difficulties administering chemotherapy after open esophagectomy have led some investigators to conclude that neoadjuvant therapy is the only option. ${ }^{20,21}$

In our center, we perform minimally invasive esophagectomy (MIE), regardless of tumor stage or the use of neoadjuvant therapy. We hypothesized that patients treated with primary MIE followed by adjuvant therapy would have comparable oncologic outcomes compared with patients treated with neoadjuvant therapy followed by MIE. Because treatment assignment was not random, we adjusted for large differences in observed covariates between groups using a propensity score and examined whether primary MIE followed by adjuvant chemotherapy influenced survival compared with propensity-matched patients treated with neoadjuvant therapy.

\section{PATIENTS AND METHODS}

We reviewed all patients with clinical stage II or higher EAC treated with MIE (January 1, 1997-July 31, 2009; $\mathrm{N}=375$ ). Our approach to MIE has been previously described. ${ }^{22-25}$ Eight stage IVb patients who underwent MIE for bleeding and/or perforation and were excluded. Pretreatment nodal and distant metastases were evaluated with computed tomography $(\mathrm{CT})$ scan $(\mathrm{n}=339)$, positron-emission testing (PET) scan $(n=110)$, endoscopic ultrasound (EUS) $(n=244)$, and/or laparoscopic staging $(n=150)$. Because the number of clinically positive nodes were not routinely reported, clinical stage was assigned using the American Joint Committee on Cancer (AJCC) sixth edition. ${ }^{26}$ Definitive pretreatment clinical stage was assigned only if tumor depth was assessed by EUS; when EUS was not performed or available for review, overall pretreatment clinical stage was considered undocumented unless CT or PET revealed celiac node involvement, which is stage IVa disease, according to the AJCC.

\section{Propensity-Score Matching}

We generated propensity scores to determine the probability of treatment assignment to either group. The dependent variable in the logistic regression model was treatment assignment (neoadjuvant [E-] or primary MIE [E+]); the independent variables were clinically relevant pretreatment covariates (Table 1). Patients were then matched without replacement and without ties. Due to perfect separation between neoadjuvant and primary MIE, patients with nonelective surgery $(\mathrm{n}=11)$, other metastatic tumor $(\mathrm{n}=2)$, cervical mass location $(\mathrm{n}=1)$, or clinical tumor stage T1 $(n=8)$ were excluded from propensity scoring. Six patients were excluded because of missing data in 1 or more propensity scoring variables. Propensity scores were generated for 339 patients representing the final unmatched cohort (Table 2).
We took each exposure patient ([E+] primary MIE), 1 at a time, and found all control patients ([E-] neoadjuvant therapy followed by MIE) still in the matching pool whose propensity scores were within 0.05 of the exposure patient's score. If a suitable match was not available, the patient was not included in the matched dataset. Matching was repeated several times with different random number generator seeds to ensure that matching balance and final outcome analysis produced similar, stable results each time, regardless of random seed. (Data not shown.)

Before propensity-score matching, neoadjuvant patients were significantly younger and more likely to have daily alcohol use and pretreatment complaints of dysphagia, whereas patients with primary MIE had higher age-adjusted Charlson Comorbidity Index $(\mathrm{CCI})$ scores $(P=.025) .{ }^{27} \mathrm{At}$ least 1 comorbid condition was present in $56 \%$ of patients (range, 1-6; $\mathrm{n}=190$ out of 339) and 2 or more comorbid conditions were present in $22 \%(\mathrm{n}=74$ out of 339$)$. EUS showing pretreatment invasion into the muscularis propria (T2) was more common in the patients with primary MIE; they were also less likely to have adventitial (T3) invasion. Pretreatment clinical stage III to IVa was more common in the neoadjuvant cohort, but there were no differences in pretreatment nodal metastasis rates, tumor location, or grade (Table 2).

Ninety-seven closely matched pairs $(\mathrm{n}=194)$ were generated using Stata. ${ }^{28}$ Before matching, overall mean percent bias was $15.2 \%$ $(P<.001$ for differences between the cohorts). After matching, overall mean percent bias was $6 \%(P=.973)$, with $<10 \%$ bias for most variables and $<20 \%$ in all variables except history of smoking (Table 3 ). Age (median, 64 years for both; $P=.895$ ) and age-adjusted CCI score (2 vs $1.5 ; P=.2757)$ were similar between matched and unmatched patients; median survival was 20.3 versus 23.65 months $(P=.1094)$ and recurrence rates were $58 \%$ versus $53 \%(P=.377)$, respectively. The area under the receiver operator characteristic (ROC) curve (c-index) was 0.778 , indicating very good discrimination.

\section{Statistical Analysis}

Statistical analysis was performed using Stata 13, summarizing descriptive statistics with frequencies and percentages for categorical variables and median with interquartile range (IQR) for continuous variables. Variables associated with adjuvant therapy were assessed using logistic regression. Data missingness was random. Survival time was defined as time from esophagectomy to date of last living contact or death. Time to recurrence was defined as time from esophagectomy to last clinical evaluation for recurrence. Survival curves for matched cohorts were compared using log-rank test for equality of survivor functions. Hazard ratios for death were calculated using stratified (matched) Cox proportional hazards regression with clustered standard errors for pairs after controlling for age, sex, body mass index, smoking, age-adjusted CCI, and final AJCC 7 pathologic stage. ${ }^{29}$

\section{RESULTS \\ Treatment and Perioperative Outcomes for the Entire Cohort}

Initial therapy was MIE in 156 patients (46\%) and neoadjuvant therapy in 183 patients $(54 \% ; 51 \%$ chemotherapy alone and $49 \%$ chemoradiation). Over the 12 -year timeframe, neoadjuvant therapy use ranged from $30 \%$ to $70 \%$ per year, but has been stable between $40 \%$ and $50 \%$ since 2006. Cisplatin (76\%), 5-flourouracil (61\%), and paclitaxel ( $44 \%$ ) were used in combination in $32 \%$ of patients; $19 \%$ received the combination of 5 -flourouracil and cisplatin. Carboplatin (19\%), oxaliplatin (2\%), irinotecan $(23 \%)$, docetaxel $(6 \%)$, and epirubicin $(6 \%)$ were used in combination in the remaining patients. Median delivered radiation dose was 5040 cGray (IQR, 4500-5040 cGy). 
TABLE 1. Variables used for propensity scoring

\begin{tabular}{llll}
\hline Age & Final pretreatment clinical stage & Surgeon* & Any neurologic disorder \\
Sex & Final pretreatment T stage & Urgency of operation & History of cerebrovascular accident \\
Smoking history & Final pretreatment N stage & Reoperation & History of transient ischemic attack \\
Body mass index & Final pretreatment M stage & History of myocardial infarction & Malignancy other than current \\
Daily alcohol use & History of Barrett's esophagus & Coronary artery bypass graft & Metastatic cancer \\
Gastroesophageal reflux disease & Location of mass in esophagus & History of CHF & Liver disease \\
Dysphagia & Pretreatment tumor grade & Renal insufficiency or failure & Vascular disease \\
& & Diabetes mellitus & Pulmonary disease \\
\hline
\end{tabular}

CHF, Congestive heart failure. *James D. Luketich, MD.

A median of 21 lymph nodes were identified in the pathologic specimen after MIE, with slightly fewer lymph nodes examined in the neoadjuvant group. R0 resection, defined as compete resection of all gross and microscopic disease, and negative mucosal margins were achieved in $97 \%$ of patients $(\mathrm{n}=329)$, with no difference between groups. Neoadjuvant therapy specimens were more likely to be node-negative, have lower pathologic grade, smaller tumor size, and less likely to have angiolymphatic invasion at resection, whereas primary MIE specimens were more likely to have tumor cells at the radial margin (Table 4).

Median survival for the overall cohort was 22.6 months (IQR, 9.9-47.8).

\section{Pathologic Complete Response (PCR) and Prognosis}

PCR $(13.1 \%)$ differed significantly between patients treated with chemoradiation $(25 \% ; 22$ out of 88 patients) and chemotherapy alone $(2.1 \% ; 2$ out of 94 patients; $P<.001)$. Median survival after neoadjuvant chemoradiation $(\mathrm{n}=88)$, was 36.2 versus 18.2 months when PCR occurred (log-rank test $P=.02$ ). When survival in PCR patients was compared with the combined group of patients with residual tumor after neoadjuvant therapy and primary MIE, a survival advantage after neoadjuvant therapy was not realized, although there was a trend toward significance (median, 39.5 vs 21.9 months; log-rank test $P=.09$ ).

\section{Administration of Adjuvant Therapy in the Overall Cohort}

Data regarding adjuvant therapy was available for $311 \mathrm{pa}-$ tients overall ( $92 \%$ ) and 178 matched patients (92\%). Adjuvant chemotherapy was administered to $49 \%$ of neoadjuvant (86 out of 176) and $49 \%$ of primary MIE patients (66 out of $135 ; P=1.000)$. Factors associated with adjuvant therapy included age at operation $(P<.001)$ and age-adjusted CCI $(P<.001)$. Pathologic tumor factors associated with adjuvant therapy in univariate analysis include AJCC 7 pathologic stage II or greater $(P<.001),{ }^{29}$ T3 depth of invasion $(P=.002)$, presence and increasing number of pathologically positive nodes $(P<.001$ for both), viable tumor at esophagectomy $(P=.001)$, larger pathologic tumor size $(P<.001)$, angiolymphatic invasion $(P=.047)$, positive circumferential $(P=.005)$ and mucosal $(P=.01)$ margins, pathologic grade $(P<.001)$, and R0 resection $(P=.100)$. After adjusting for all significant variables, age younger than 70 years $(P=.001)$ and CCI score $<3(P=.014)$ were the only significant predictors of exposure to adjuvant therapy. The cstatistic for the logistic model was 0.778 , indicating good discrimination for predicting adjuvant therapy after MIE.

\section{Propensity-Score Matched Recurrence and Survival Analysis}

In the 97 propensity-score matched pairs, median time to last clinical follow-up or death was 20.3 months (IQR, 9.9-43.9). Median overall survival was 18.7 months (IQR, 9-36), with no difference between propensity-score matched groups (Figure $1, A$ ) (log-rank test $P=.679$ ). To account for paired data and censoring, multivariate clustered Cox regression was performed. After adjusting for age, sex, body mass index, smoking, age-adjusted CCI score, and AJCC 7 pathologic stage, ${ }^{29}$ primary MIE was not associated with significantly different hazard for death (hazard ratio, $0.83 ; 95 \%$ confidence interval, $0.60-1.16)$. Pathologic stage was a significant prognostic variable $(P=.006)$.

In the 97 propensity-score matched pairs, 113 patients $(58 \%)$ had developed recurrence at a median follow-up of 10.1 months (IQR, 4.1-21.1) with no difference between propensity score-matched groups in either the proportion with recurrence (neoadjuvant 55 out of 97 [57\%] vs primary MIE 58 out of 97 [60\%], respectively; $P=.771)$ or time to recurrence (mean 14.9 vs 15.8 months, respectively; logrank test $P=.924$ ) (Figure 1, $B$ ). When identified, recurrence was distant in $76 \%$ and $78 \%$ of neoadjuvant ( 42 out of 55 ) and primary MIE patients (45 out of $58 ; P=.118$ ), respectively. Chemotherapy alone showed a trend toward higher recurrence rates compared with chemoradiation (64\% [38 out of 59 patients] vs $45 \%$ [ 17 out of 38 patients]; $P=.063$ ), with $76 \%$ of patients (29 out of 38 ) and $77 \%$ of patients (13 out of 17) presenting with distant metastasis, respectively.

\section{Administration of Adjuvant Therapy in the Propensity-Score Matched Cohort}

In the propensity-score matched cohort, adjuvant therapy was given to $47 \%$ of the neoadjuvant cohort (43 of 92 with 
TABLE 2. Pretreatment patient demographics and characteristics for the entire cohort comparing patients assigned to neoadjuvant therapy with those assigned to minimally invasive esophagectomy (MIE) as primary therapy

\begin{tabular}{|c|c|c|c|c|}
\hline \multirow[b]{3}{*}{ Pretreatment clinical characteristic } & \multicolumn{3}{|c|}{ Before matching in propensity-scored patients } & \multirow[b]{3}{*}{$P$ value } \\
\hline & Primary MIE & Neoadjuvant therapy & All patients & \\
\hline & $(\mathbf{n}=156)$ & $(\mathbf{n}=183)$ & $(\mathbf{n}=339)$ & \\
\hline Age, y & $67(59-75)$ & $63(54-70)$ & $64(56-72)$ & $<.001$ \\
\hline Body mass index & $27.4(24.8-30.9)$ & $27.2(23.6-31.1)$ & $27.3(24.4-31)$ & .472 \\
\hline Male sex & 131 & 157 & 288 & .651 \\
\hline Surgeon* & 99 & 138 & 237 & .018 \\
\hline History of smoking ( $>100$ cigarettes in lifetime) & 111 & 144 & 255 & .130 \\
\hline Daily alcohol use & 17 & 36 & 53 & .035 \\
\hline Pretreatment dysphagia & 115 & 159 & 274 & .002 \\
\hline Documented history of gastroesophageal reflux disease & 107 & 115 & 222 & .303 \\
\hline Histologically confirmed Barrett's esophagus & 92 & 96 & 188 & .273 \\
\hline Prior esophageal operation & 12 & 8 & 20 & .249 \\
\hline \multicolumn{5}{|l|}{ Comorbid illnesses } \\
\hline Overall age-adjusted Charlson Comorbidity Index Score & $3(0-4)$ & $1(0-4)$ & $2(0-4)$ & .025 \\
\hline Myocardial infarction or revascularization & 37 & 35 & 72 & .351 \\
\hline Coronary bypass graft or stent & 27 & 22 & 49 & .215 \\
\hline Congestive heart failure & 5 & 4 & 9 & .738 \\
\hline Peripheral vascular disease & 9 & 12 & 21 & .824 \\
\hline Creatinine $\geq 2.0$ or need for hemodialysis & 1 & 2 & 3 & 1.000 \\
\hline Diabetes requiring medical therapy & 29 & 23 & 52 & .133 \\
\hline Pulmonary disease & 30 & 38 & 68 & .786 \\
\hline Neurological disorder & 13 & 8 & 21 & .175 \\
\hline Transient ischemic attack & 10 & 5 & 15 & .117 \\
\hline Cerebrovascular accident & 5 & 4 & 9 & .738 \\
\hline Malignancy other than esophageal cancer & 10 & 13 & 23 & .832 \\
\hline Liver dysfunction (child's class A, B, or C) & 2 & 3 & 5 & 1.000 \\
\hline \multicolumn{5}{|l|}{ Pretreatment tumor-specific variables } \\
\hline Pretreatment location of mass by endoscopy & & & & 1.000 \\
\hline Middle esophagus & 1 & 1 & 2 & \\
\hline Lower esophagus & 48 & 56 & 104 & \\
\hline GEJ/cardia & 107 & 126 & 233 & \\
\hline Pretreatment tumor grade & & & & .113 \\
\hline Well differentiated & 15 & 6 & 21 & \\
\hline Moderately differentiated & 43 & 58 & 101 & \\
\hline Poorly differentiated & 75 & 91 & 166 & \\
\hline Not reported & 23 & 28 & 51 & \\
\hline Final pretreatment tumor depth* & & & & .002 \\
\hline T2 (into muscularis propria) & 29 & 19 & 48 & \\
\hline $\mathrm{T} 3$ (into adventitia) & 74 & 119 & 193 & \\
\hline T4a (into adjacent, resectable structures) & 2 & 6 & 8 & \\
\hline Pretreatment $\mathrm{T}$ stage unknown & 51 & 39 & 90 & \\
\hline Final pretreatment nodal status $\dagger$ & & & & .919 \\
\hline N0 (no clinically positive nodes) & 36 & 43 & 79 & \\
\hline N1 (clinically positive nodes) & 117 & 135 & 252 & \\
\hline $\mathrm{N}$ stage unknown & 3 & 5 & 8 & \\
\hline Final pretreatment clinical stage & & & & $<.001$ \\
\hline Stage IIa & 3 & 19 & 22 & \\
\hline Stage IIb & 27 & 14 & 41 & \\
\hline Stage III & 69 & 91 & 160 & \\
\hline Stage IVa (celiac node involvement) & 6 & 26 & 32 & \\
\hline Pretreatment stage undocumented & 51 & 33 & 84 & \\
\hline
\end{tabular}

Values are presented as n or median (interqartile range). MIE, Minimally invasive esophagectomy; GEJ, gastroesophageal junction. *Pretreatment tumor depth assigned only for those patients with a pretreatment endoscopic ultrasound documenting depth of invasion into the esophageal wall. Pretreatment $\mathrm{T}$ stage was not assigned based on computed tomography signs or symptoms. †Pretreatment node status assigned based on computed tomography scan, positron emission tomography scan, endoscopic ultrasound, and/or laparoscopic staging. 
TABLE 3. Comparison of clinical variables between groups after propensity score assignment and after propensity matching

Before matching in

propensity-scored patients

\begin{tabular}{|c|c|c|c|c|c|c|c|}
\hline \multirow[b]{2}{*}{$\begin{array}{c}\text { Pretreatment clinical } \\
\text { characteristics }\end{array}$} & \multicolumn{3}{|c|}{ propensity-scored patients } & \multicolumn{2}{|c|}{ Matched cohort } & \multirow[b]{2}{*}{$\%$ Bias } & \multirow[b]{2}{*}{$\begin{array}{l}\% \text { Bias } \\
\text { reduction }\end{array}$} \\
\hline & $\frac{\text { Primary MIE }}{(\mathrm{n}=156)}$ & $\begin{array}{c}\begin{array}{c}\text { Neoadjuvant } \\
\text { therapy }\end{array} \\
(n=183)\end{array}$ & $\%$ Bias & $\frac{\text { Primary MIE }}{(\mathrm{n}=97)}$ & $\begin{array}{c}\begin{array}{c}\text { Neoadjuvant } \\
\text { therapy }\end{array} \\
(n=97)\end{array}$ & & \\
\hline Mean age, y & 66.4 & 61.7 & 44 & 64.1 & 63.5 & 5.8 & 86.9 \\
\hline Mean body mass index & 28.6 & 28.1 & 7.8 & 29.4 & 29.3 & 1.9 & 75.6 \\
\hline Male sex & 84 & 86 & -5.1 & 82.5 & 82.5 & 0 & 100 \\
\hline Surgeon* & 63.5 & 75.4 & -26.2 & 74.2 & 66 & 18 & 31 \\
\hline $\begin{array}{l}\text { History of smoking ( }>100 \text { cigarettes in } \\
\text { lifetime) }\end{array}$ & 71.2 & 78.7 & -17.4 & 81.4 & 71.1 & 23.8 & -36.8 \\
\hline Daily alcohol use & 10.9 & 19.7 & -24.5 & 13.4 & 9.3 & 11.5 & 53 \\
\hline Pretreatment dysphagia & 73.7 & 86.9 & -33.5 & 85.6 & 84.5 & 2.6 & 92.2 \\
\hline $\begin{array}{l}\text { Documented history of gastroesophageal } \\
\text { reflux disease }\end{array}$ & 68.6 & 62.8 & 12.1 & 65 & 68 & -6.5 & 46.2 \\
\hline Histologically confirmed Barrett's esophagus & 59 & 52.5 & 13.1 & 55.7 & 60.8 & -10.4 & 20.9 \\
\hline Prior esophageal operation & 7.7 & 4.4 & 13.9 & 4.1 & 4.1 & 0 & 100 \\
\hline \multicolumn{8}{|l|}{ Charlson-defined comorbid illness } \\
\hline $\begin{array}{l}\text { History of myocardial infarction or } \\
\text { revascularization }\end{array}$ & 23.7 & 19.1 & 11.2 & 19.6 & 20.6 & -2.5 & 77.6 \\
\hline History of coronary bypass graft or stent & 17.3 & 12 & 14.9 & 14.4 & 14.4 & 0 & 100 \\
\hline History of congestive heart failure & 3.2 & 2.2 & 6.3 & 4.1 & 3.1 & 6.3 & -1.1 \\
\hline History of peripheral vascular disease & 5.8 & 6.6 & -3.3 & 5.2 & 5.2 & 0 & 100 \\
\hline Creatinine $\geq 2.0$ or need for hemodialysis & 0.6 & 1.1 & -4.9 & 1 & 1 & 0 & 100 \\
\hline Diabetes requiring medical therapy & 18.6 & 12.6 & 16.6 & 16.5 & 17.5 & -2.8 & 82.9 \\
\hline History of pulmonary disease & 19.2 & 20.8 & -3.8 & 20.6 & 19.6 & 2.6 & 32.8 \\
\hline History of neurological disorder & 8.3 & 4.4 & 16.2 & 6.2 & 7.2 & -4.2 & 74 \\
\hline History of transient ischemic attack & 6.4 & 2.7 & 17.6 & 4.1 & 4.1 & 0 & 100 \\
\hline History of cerebrovascular accident & 3.2 & 2.2 & 6.3 & 1 & 3.1 & -12.7 & -102.3 \\
\hline $\begin{array}{l}\text { History of malignancy other than } \\
\text { esophageal cancer }\end{array}$ & 6.4 & 7.1 & -2.8 & 7.2 & 5.2 & 8.2 & -197.3 \\
\hline $\begin{array}{l}\text { History of liver dysfunction (child's class } \\
\text { A, B or C) }\end{array}$ & 1.3 & 1.6 & -3 & 2.1 & 1 & 8.6 & -188.5 \\
\hline \multicolumn{8}{|l|}{ Pretreatment tumor-specific variables } \\
\hline \multicolumn{8}{|l|}{ Pretreatment location of mass by endoscopy } \\
\hline Middle esophagus & 0.64 & 0.55 & 1.2 & 1 & 1 & 0 & 100 \\
\hline Lower esophagus & 30.8 & 30.6 & 0.4 & 29.9 & 30.9 & -2.2 & -513.1 \\
\hline GEJ/cardia & 68.6 & 68.9 & -0.6 & 69.1 & 68 & 2.2 & -292.4 \\
\hline \multicolumn{8}{|l|}{ Pretreatment tumor grade } \\
\hline Well differentiated & 9.6 & 3.3 & 25.9 & 7.2 & 5.2 & 8.4 & 67.5 \\
\hline Moderately differentiated & 27.6 & 31.7 & -9 & 28.9 & 33 & -9 & 0.1 \\
\hline Poorly differentiated & 48.1 & 49.7 & -3.3 & 50.5 & 52.6 & -4.1 & -25 \\
\hline Not reported & 14.7 & 15.3 & -1.6 & 13.4 & 9.3 & 11.5 & -640.4 \\
\hline \multicolumn{8}{|c|}{ Individual and overall pretreatment clinical stage variables } \\
\hline \multicolumn{8}{|c|}{ Final pretreatment tumor depth $\dagger$} \\
\hline T2 (into muscularis propria) & 18.6 & 10.4 & 23.4 & 12.4 & 15.5 & -8.8 & 62.3 \\
\hline T3 (into adventitia) & 47.4 & 65 & -35.9 & 55.7 & 55.7 & 0 & 100 \\
\hline T4a (into adjacent, resectable structures) & 1.3 & 3.3 & -13.4 & 2.1 & 2.1 & 0 & 100 \\
\hline Pretreatment $\mathrm{T}$ stage unknown & 32.7 & 21.3 & 25.8 & 29.9 & 26.8 & 7 & 72.8 \\
\hline \multicolumn{8}{|l|}{ Final pretreatment nodal statusł } \\
\hline N0 (no clinically positive nodes) & 23.1 & 23.5 & -1 & 21.7 & 15.5 & 14.6 & -1371.5 \\
\hline N1 (clinically positive nodes) & 75 & 73.8 & 2.8 & 76.3 & 81.4 & -11.8 & -319.2 \\
\hline $\mathrm{N}$ stage unknown & 1.9 & 2.7 & -5.4 & 2.1 & 3.1 & -6.8 & -27.4 \\
\hline
\end{tabular}




\begin{tabular}{|c|c|c|c|c|c|c|c|}
\hline \multirow[b]{2}{*}{$\begin{array}{c}\text { Pretreatment clinical } \\
\text { characteristics }\end{array}$} & \multicolumn{3}{|c|}{$\begin{array}{c}\text { Before matching in } \\
\text { propensity-scored patients }\end{array}$} & \multicolumn{2}{|c|}{ Matched cohort } & \multirow[b]{2}{*}{$\%$ Bias } & \multirow[b]{2}{*}{$\begin{array}{l}\% \text { Bias } \\
\text { reduction }\end{array}$} \\
\hline & $\frac{\text { Primary MIE }}{(n=156)}$ & $\begin{array}{c}\begin{array}{c}\text { Neoadjuvant } \\
\text { therapy }\end{array} \\
(n=183)\end{array}$ & $\%$ Bias & $\frac{\text { Primary MIE }}{(\mathrm{n}=97)}$ & $\begin{array}{c}\begin{array}{c}\text { Neoadjuvant } \\
\text { therapy }\end{array} \\
(n=97)\end{array}$ & & \\
\hline \multicolumn{8}{|l|}{ Final pretreatment clinical stage } \\
\hline Stage IIa & 1.9 & 10.4 & -35.7 & 3.1 & 1 & -9.4 & 75.6 \\
\hline Stage IIb & 17.3 & 7.7 & 29.4 & 10.3 & 13.4 & -9.4 & 68 \\
\hline Stage III & 44.2 & 49.7 & -11 & 50.5 & 53.6 & -6.2 & 43.7 \\
\hline Stage IVa (celiac node involvement) & 3.9 & 14.2 & -36.7 & 6.2 & 5.2 & 3.6 & 90.1 \\
\hline Pretreatment stage undocumented & 32.7 & 18 & 34.1 & 29.9 & 26.8 & 7.2 & 78.9 \\
\hline
\end{tabular}

Values are given as \%, unless otherwise noted. MIE, Minimally invasive esophagectomy; GEJ, gastroesophageal junction. *James D. Luketich, MD. †Pretreatment tumor depth assigned only for those patients with a pretreatment endoscopic ultrasound documenting depth of invasion into the esophageal wall. Pretreatment $\mathrm{T}$ stage was not assigned based on computed tomography signs or symptoms. ŁPretreatment nodal status assigned based on computed tomography scan, positron emission tomography scan, endoscopic ultrasound, and/or laparoscopic staging.

available data) compared with $56 \%$ of primary MIE patients (48 of 86 with available data; $P=.235$ ). Since 2006, adjuvant therapy was given to $65 \%$ of patients (41 out of 63 patients) in the matched pairs with pathologic stage IIb or greater, including 70\% (33 out of 47) of primary MIE and $50 \%$ (8 out of 16$)$ of neoadjuvant patients $(P=.224)$.

\section{Accuracy of Clinical Staging}

Clinical staging was inaccurate in $35 \%$ of primary MIE patients who had complete clinical staging (EUS plus CT and/or PET-CT) before surgery (37 out of 105 patients). Eighteen patients were upstaged; 3 patients assigned to clinical stage IIa were stage IIIb $(n=1)$ and IIIc $(n=2)$ on final pathology. Thirteen patients assigned to clinical stage IIb were IIIa $(n=7)$, IIIb $(n=3)$, and IIIc $(n=3)$ on final pathology. Two patients with clinical stage III had resectable solitary liver metastasis discovered at esophagectomy and were assigned pathologic stage IV. Nineteen were downstaged after resection; 4 patients assigned clinical stage IIb were changed to stage IIa $(n=3)$ and Ib $(n=1)$ on final pathology. Fourteen patients assigned clinical stage III were reassigned to $\mathrm{Ib}(\mathrm{n}=1)$, IIa $(\mathrm{n}=4)$, and IIb $(\mathrm{n}=9)$ on final pathology. One patient assigned to clinical stage IVa was downstaged to stage IIb.

\section{DISCUSSION}

We performed a propensity-score matched analysis comparing neoadjuvant therapy followed by MIE to MIE as primary treatment for locally advanced EAC. We found that patients treated with primary MIE followed by adjuvant therapy based on pathologic stage did not differ significantly in terms of overall survival or recurrent disease compared with patients treated with neoadjuvant therapy. In addition, primary MIE allowed for accurate staging compared with clinical staging in nearly one-third of patients. These findings suggest that primary MIE followed by adjuvant therapy in patients with resectable locally advanced EAC is a reasonable alternative to treating all patients with neoadjuvant therapy. By allowing accurate stage assignment through pathologic assessment of the primary tumor and the lymph nodes, MIE followed by adjuvant chemotherapy in appropriate patients could improve prognostication and potentially minimize the risk of overtreatment in patients with early stage disease.

\section{Examining the Role of Neoadjuvant Therapy}

There is little debate about the role of surgery in the management of $\mathrm{EAC}^{30}$; analyses of large population-based datasets and single-center reports clearly demonstrate improved survival in patients treated with a trimodality approach compared with chemoradiation alone. ${ }^{31,32}$ The role for neoadjuvant therapy for EAC continues to be refined, in part because most of the randomized controlled trials comparing neoadjuvant therapy with surgery alone include both squamous and adenocarcinoma histologies. A recent meta-analysis suggested that patients with adenocarcinoma experience a survival benefit with neoadjuvant chemo(radio)therapy compared with surgery alone, ${ }^{2,11}$ as did the Chemoradiotherapy for Oesophageal Cancer followed by Surgery Study (CROSS) trial, ${ }^{33}$ which included adenocarcinoma histologic subtype for $75 \%$ of patients. In this study, the median survival for adenocarcinoma was approximately 28 months with surgery alone compared with 48 months after chemoradiotherapy followed by surgery $(P=.049)$. However, when adjusted for baseline covariates, including sex, clinical $\mathrm{N}$ stage, and performance status, the $25.9 \%$ reduction in hazard for death in patients with adenocarcinoma treated with neoadjuvant chemoradiotherapy compared with surgery alone was not statistically significant. ${ }^{33}$ In comparison, there was a nearly $58 \%$ reduction in hazard of death with chemoradiotherapy in the squamous histology group $(P=.007)$. Although interpreted as a strong statement in favor of neoadjuvant chemoradiotherapy for all esophageal cancer patients, the survival benefit in the adenocarcinoma subset is less compelling, particularly when adjusted for other survival predictors. Importantly, as with other studies, 
TABLE 4. Pathologic outcomes after minimally invasive esophagectomy (MIE) comparing neoadjuvant therapy followed by MIE compared with MIE as primary treatment in propensity-matched and overall patient cohorts

\begin{tabular}{|c|c|c|c|c|c|c|c|}
\hline \multirow[b]{3}{*}{ Pathologic finding } & \multicolumn{3}{|c|}{ Propensity-matched cohort } & \multicolumn{4}{|c|}{ Overall cohort } \\
\hline & $\begin{array}{l}\text { Neoadjuvant } \\
\text { therapy }\end{array}$ & Primary MIE & & $\begin{array}{l}\text { Neoadjuvant } \\
\text { therapy }\end{array}$ & $\begin{array}{l}\text { Primary } \\
\text { MIE }\end{array}$ & $\begin{array}{c}\text { Total } \\
\text { patients }\end{array}$ & \\
\hline & $(\mathbf{n}=\mathbf{9 7})$ & $(\mathbf{n}=\mathbf{9 7})$ & $P$ value & $(\mathbf{n}=183)$ & $(n=156)$ & $(\mathbf{n}=339)$ & $P$ value \\
\hline Number of lymph nodes examined & $20(15-27)$ & $25(17-33)$ & .034 & $20(14-27)$ & $24(16-32)$ & $21(15-29)$ & .003 \\
\hline Number of positive lymph nodes & $1(0-5)$ & $3(1-9)$ & .002 & $1(0-4)$ & $3(1-7)$ & $2(0-6)$ & .002 \\
\hline Node-negative at resection & $46(47)$ & $20(21)$ & .001 & $79(43)$ & $36(23)$ & $122(34)$ & .001 \\
\hline Pathologic grade & & & $<.001$ & & & & $<.001$ \\
\hline Well differentiated & $1(1)$ & $1(1)$ & & $1(0.55)$ & $4(2)$ & $4(1.2)$ & \\
\hline Moderately differentiated & $35(36)$ & $35(36)$ & & $66(36)$ & $53(34)$ & $119(35)$ & \\
\hline Poorly differentiated & $40(41)$ & $61(63)$ & & $78(43)$ & $99(64)$ & $177(52)$ & \\
\hline No residual tumor & $15(16)$ & $0(0)$ & & $30(16)$ & $0(0)$ & $30(9)$ & \\
\hline Unable to determine* & $6(6)$ & $0(0)$ & & $8(4)$ & $1(0.6)$ & $9(3)$ & \\
\hline Tumor size, $\mathrm{cm}$ & $3.1(0.5-5.3)$ & $5(3.5-6.5)$ & $<.001$ & $3.5(0.3-5.2)$ & $5(3.5-6.5)$ & $4(2.3-6)$ & $<.001$ \\
\hline Angiolymphatic invasion $\dagger$ & 40/81 (49) & 76/93 (82) & $<.001$ & $80(52)$ & $115(76)$ & $195(64)$ & $<.001$ \\
\hline Mucosal margins negative for tumor involvement & $96(99)$ & $91(94)$ & .118 & $180(98)$ & $149(96)$ & $329(97)$ & .196 \\
\hline Tumor involving radial margin & $7(7)$ & $13(13)$ & .237 & $13(7)$ & $22(14)$ & $35(10)$ & .048 \\
\hline R0 resection $\ddagger$ & $96(99)$ & $91(94)$ & .118 & $180(98)$ & $149(96)$ & $329(97)$ & .196 \\
\hline $\begin{array}{l}\text { American Joint Committee on Cancer } 7 \\
\text { pathologic stage }\end{array}$ & & & $<.001$ & & & & $<.001$ \\
\hline Stage 0: Complete pathologic response $\S$ & $15(15.5)$ & NA & & $24(13)$ & NA & $24(7.1)$ & \\
\hline Stage 1A & $9(9)$ & $0(0)$ & & $10(5.5)$ & $0(0)$ & $10(3)$ & \\
\hline Stage 1B & $5(5)$ & $4(4)$ & & $12(6.6)$ & $8(5)$ & $20(6)$ & \\
\hline Stage IIA & $4(4)$ & $5(5)$ & & $7(4)$ & $9(6)$ & $16(5)$ & \\
\hline Stage IIB & $15(16)$ & $15(16)$ & & $37(20)$ & $30(19)$ & $67(20)$ & \\
\hline Stage IIIA & $18(19)$ & $18(19)$ & & $39(21)$ & $34(22)$ & 73 (22) & \\
\hline Stage IIIB & $11(11)$ & $20(21)$ & & $21(12)$ & $31(20)$ & $52(15)$ & \\
\hline Stage IIIC & $19(20)$ & $33(34)$ & & $30(16)$ & $42(27)$ & $72(21)$ & \\
\hline Stage IV & $1(1)$ & $2(2)$ & & $3(2)$ & $2(1)$ & $5(1.5)$ & \\
\hline
\end{tabular}

Values are presented as median (interquartile range) or $\mathrm{n}(\%) . N A$, Not applicable; MIE, minimally invasive esophagectomy. *Tumor grade unable to be determined in patients with no residual tumor or only scattered residual cells. †Angiolymphatic invasion was not reported in the final pathology on all patients. $\ddagger \mathrm{R} 0$ resection is defined as a microscopically margin-negative resection, in which no gross or microscopic tumor remains in the primary tumor bed; R1 resection is defined as removal of all macroscopic disease but microscopic resection margins are positive. Radial margin involvement with tumor is not considered an R1 resection unless the tumor was adherent to adjacent structures (eg, liver, diaphragm, pleura, pericardium, or prevertebral fascia) and dissected free. R2 indicates gross residual tumor (primary tumor, regional nodes, and macroscopic margins) but does not indicate metastatic disease to distant organs. §̊ndicates no residual cancer within the esophagus or metastatic disease in the examined lymph nodes.

adjuvant therapy was not given to the surgery alone groups, meaning that patients with metastatic disease to the lymph nodes did not receive any systemic therapy. In contrast, adjuvant therapy was given to $65 \%$ of our propensity-score matched patients with IIB or greater pathologic stage and may explain the similar survival between groups in our study.

A major goal of neoadjuvant therapy is to achieve a complete pathologic response, with no residual disease in the esophagus or in the surrounding lymph nodes. However, in most studies, pathologic complete response is only found in $15 \%$ to $25 \%$ of patients treated with chemoradiation. ${ }^{33-37}$ The most recent randomized trial (the CROSS trial) had a $23 \%$ complete response rate after chemoradiotherapy in the adenocarcinoma group. ${ }^{33}$ In patients treated with neoadjuvant chemotherapy without radiation, response rates range from $0 \%$ to $5 \% .{ }^{10,38,39}$ The complete response rate in this study is consistent with the reported literature. When we examined the patients treated with chemoradiation, we did see a significant improvement in survival associated with a PCR compared with chemoradiation patients without a complete response. This is also consistent with the reported literature. ${ }^{40}$ We did not find improved survival with PCR compared with all patients with residual tumor at esophagectomy (including patients who did not have PCR after neoadjuvant and patients treated with primary MIE). Although potentially explained by insufficient power related to small numbers of PCR, the lack of survival advantage might be due to the delivery of adjuvant therapy to a high percentage of patients with nodal involvement after MIE as primary therapy. This possibility requires prospective validation in a randomized controlled trial.

In addition to optimizing survival, objective assessment of risks and benefits must be considered when analyzing the role for neoadjuvant therapy. Schneider and colleagues ${ }^{41}$ evaluated response to therapy and survival and found that patients in whom disease was stable (ie, 

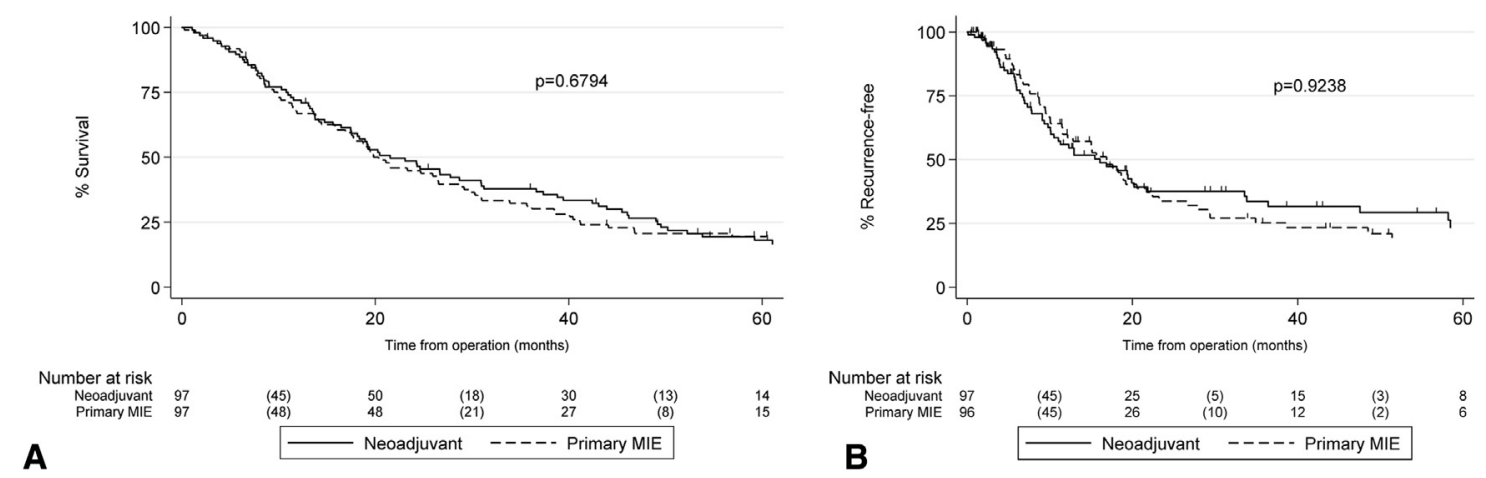

FIGURE 1. A, Overall survival after minimally invasive esophagectomy (MIE). Propensity-matched comparison of neoadjuvant therapy followed MIE compared with MIE as primary therapy. The numbers in parenthesis are the number of failure events (deaths) between each time point. B, Propensitymatched comparison of the percentage of patients free of recurrence over time after MIE. Neoadjuvant therapy followed by MIE versus MIE as primary therapy. The numbers in parentheses are the number of failure events (deaths) between each time point. In the primary MIE cohort, 1 patient had an R2 resection and persistent disease and was, therefore, never rendered free of disease. Number at risk, therefore, is 96 at time 0.

nonresponders) or who presented evidence for progression of disease had substantially worse survival than patients with a partial or complete response. In their study, ${ }^{41}$ objective tumor regression was noted at esophagectomy in only $\sim 40 \%$ of patients; this means that $60 \%$ of patients derived no measurable benefit from the neoadjuvant therapy while being exposed to potential complications from the treatment itself. These complications were described in a systematic review of 38 articles by Courrech Staal and colleagues ${ }^{5}$; multiple grade III to IV toxicities associated with chemoradiation included dehydration $(17 \%)$, vomiting $(0 \%-16 \%)$, esophagitis $(0 \%-43 \%)$, pneumonitis $(2 \%)$, and fistula formation $(2 \%)$. Treatment-related mortality was $2.3 \%$ (29 out of 1269 patients) after the start of chemoradiation therapy but before surgical resection. ${ }^{5}$ This variability in therapeutic response and risk of associated toxicity raises the possibility that a selective approach for patients treated outside of clinical trials may be worth further study. In some cases, patients with resectable disease at diagnosis may ultimately be unable to proceed with surgical resection because of treatment-related toxicity, or worsening of underlying comorbid conditions (such as pulmonary injury from the radiation field effect). In our study, patients who were older and who had a higher CCI score were less likely to have neoadjuvant therapy before MIE and were also less likely to have adjuvant therapy than were younger patients and those with fewer comorbidities. In fact, age younger than 70 years and CCI score $<3$ were independently associated with the use of adjuvant therapy, whereas all of the tumor-specific variables (ie, the biological indicators of poor prognosis) were not. In many patients, because of toxicity risk, primary esophagectomy for resectable adenocarcinoma may be an important alternative, especially given that only $40 \%$ of patients benefit from current neoadjuvant regimens and one-third are incorrectly staged by clinical evaluation.

\section{Study Strengths and Limitations}

Several recent studies evaluating early outcomes after MIE versus open esophagectomy have shown significantly earlier return to baseline function, including global function, level of fatigue, overall physical function as measured by the physical component summary of the Short-Form 36 Health Survey (SF-36), ${ }^{42}$ quality of life measures such as pain and ability to talk, and activities of daily living. In a randomized controlled trial by Biere and colleagues, ${ }^{43}$ these differences were identified as early as 6 weeks after esophagectomy. As with the randomized trial, Parameswaran and colleagues ${ }^{44}$ noted faster resolution of physical fatigue, improving by 3 months and back to baseline at 6 months after MIE while remaining elevated after open esophagectomy. Reports of reduced motivation increased in the open group while the MIE group returned to baseline by 3 months and was below baseline (ie, more motivated) by 6 months. Finally, more MIE patients were completely independent in their instrumental activities of daily living at 3 and 6 months (53\% vs $33 \%$ and $78 \%$ vs $33 \%$, respectively). Although limited by small numbers of patients and there are no published studies to date that specifically examine the question of improved delivery of adjuvant therapy comparing open and MIE, these data suggest that the physiologic influence of MIE is of shorter duration and resolves within a timeframe (3 months) that would allow for adjuvant therapy to be considered. As noted in our study, $65 \%$ of our propensity-score matched patients with pathologic AJCC 7 stage IIB or greater (T3 depth of invasion and/or nodal metastasis in the pathologic specimen) received adjuvant therapy. The ability to deliver chemotherapy to patients with pathologically staged regionally advanced disease was likely an important factor in our finding that survival was similar between the propensityscore matched patients.

Our study is also strengthened by the use of propensityscore matching to equalize the treatment groups with regard 
to variables such as age and clinical stage, which also affect survival. The propensity score is the conditional probability of an individual to be treated given its covariates ${ }^{45}$; it is used to balance large differences in the observed covariates between 2 groups in observational studies, thereby reducing the bias in estimates of treatment effects. This allows investigators to adjust the likelihood of assignment to 1 treatment group versus another for as many covariates as possible. Using propensity-score matching, we created 2 treatment groups that were well balanced across nearly all levels of all included covariates.

Even with propensity matching, our comparisons between groups are limited by the completeness of the data and may be biased by difficult-to-measure factors, such as the thoroughness of documentation. We minimized this by performing chart review with consistent data definitions, and validation of the data by a second abstractor, but cannot completely correct for this limitation in retrospective study design. Another limitation of our study is that we are unable to discuss disease-specific mortality because the cause of death is unknown in patients who were followed-up by physicians close to their homes. As such, we compared overall survival only. Finally, the total number of patients in our study may not provide enough power to detect small but statistically significant differences in survival outcomes between groups.

Our results may not be generalizable to all surgeons and surgeon practices, particularly if they have limited experience with esophagectomy or operate in hospitals lacking the expertise to care for these patients postoperatively. In addition, the fact that our study population only includes patients who underwent MIE did not allow us to examine those patients who experienced disease progression, severe complications, and/or death while receiving neoadjuvant therapy, or who were treated with definitive chemo- and/ or chemoradiotherapy.

\section{CONCLUSIONS}

We found that primary MIE followed by adjuvant chemotherapy does not negatively influence survival compared with propensity-matched patients treated with neoadjuvant therapy for locally advanced EAC, and allowed for accurate cancer staging. Our data suggest that similar outcomes can be achieved when patients undergo MIE with curative intent, followed by adjuvant chemotherapy for disease labeled pathologic stage IIB or greater. Patients with marginally resectable disease or significant nodal involvement should still be considered for neoadjuvant therapy to improve complete resection rates. We have previously shown that minimally invasive techniques for esophagectomy can be used in a wide range of patients; improved physiologic function in this setting may facilitate delivery of adjuvant chemotherapy. ${ }^{25}$ This finding must be confirmed in prospective randomized trials before definitive conclusions can be made.
Future trials randomizing patients with EAC deemed resectable at laparoscopic staging to either neoadjuvant therapy followed by MIE or MIE followed by adjuvant therapy are needed to determine the true efficacy of this approach.

The authors thank Sunee Hempel, Megan Lunz, and Julie Ward for their assistance with data acquisition for this study. The authors also thank Shannon Wyszomierski for her excellent editorial review.

\section{References}

1. Vallbohmer D, Holscher AH, DeMeester S, DeMeester T, Salo J, Peters J, et al. A multicenter study of survival after neoadjuvant radiotherapy/chemotherapy and esophagectomy for ypTONOMOR0 esophageal cancer. Ann Surg. 2010;252: 744-9.

2. Sjoquist KM, Burmeister BH, Smithers BM, Zalcberg JR, Simes RJ, Barbour A, et al. Survival after neoadjuvant chemotherapy or chemoradiotherapy for resectable oesophageal carcinoma: an updated meta-analysis. Lancet Oncol. 2011;12: 681-92.

3. Keresztes RS, Port JL, Pasmantier MW, Korst RJ, Altorki NK. Preoperative chemotherapy for esophageal cancer with paclitaxel and carboplatin: results of a phase II trial. J Thorac Cardiovasc Surg. 2003;126:1603-8.

4. Stiles BM, Christos P, Port JL, Lee PC, Paul S, Saunders J, et al. Predictors of survival in patients with persistent nodal metastases after preoperative chemotherapy for esophageal cancer. J Thorac Cardiovasc Surg. 2010;139:387-94.

5. Courrech Staal EF, Aleman BM, Boot H, van Velthuysen ML, van Tinteren H, van Sandick JW. Systematic review of the benefits and risks of neoadjuvant chemoradiation for oesophageal cancer. Br J Surg. 2010;97:1482-96.

6. Urba SG, Orringer MB, Turrisi A, Iannettoni M, Forastiere A, Strawderman M. Randomized trial of preoperative chemoradiation versus surgery alone in patients with locoregional esophageal carcinoma. J Clin Oncol. 2001;19:305-13.

7. Walsh TN, Noonan N, Hollywood D, Kelly A, Keeling N, Hennessy TP. A comparison of multimodal therapy and surgery for esophageal adenocarcinoma. N Engl J Med. 1996;335:462-7.

8. Tepper J, Krasna MJ, Niedzwiecki D, Hollis D, Reed CE, Goldberg R, et al. Phase III trial of trimodality therapy with cisplatin, fluorouracil, radiotherapy, and surgery compared with surgery alone for esophageal cancer: CALGB 9781. J Clin Oncol. 2008;26:1086-92.

9. Kelsen DP, Ginsberg R, Pajak TF, Sheahan DG, Gunderson L, Mortimer J, et al. Chemotherapy followed by surgery compared with surgery alone for localized esophageal cancer. N Engl J Med. 1998;339:1979-84.

10. Cunningham D, Allum WH, Stenning SP, Thompson JN, Van de Velde CJ, Nicolson M, et al. Perioperative chemotherapy versus surgery alone for resectable gastroesophageal cancer. N Engl J Med. 2006;355:11-20.

11. Lee PC, Mirza FM, Port JL, Stiles BM, Paul S, Christos P, et al. Predictors of recurrence and disease-free survival in patients with completely resected esophageal carcinoma. J Thorac Cardiovasc Surg. 2011;141:1196-206.

12. Bergeron EJ, Lin J, Chang AC, Orringer MB, Reddy RM. Endoscopic ultrasound is inadequate to determine which $\mathrm{T} 1 / \mathrm{T} 2$ esophageal tumors are candidates for endoluminal therapies. J Thorac Cardiovasc Surg. 2014;147:765-71; discussion 71-3.

13. Stiles BM, Mirza F, Coppolino A, Port JL, Lee PC, Paul S, et al. Clinical T2T3N0M0 esophageal cancer: the risk of node positive disease. Ann Thorac Surg. 2011;92:491-6; discussion 6-8.

14. Young PE, Gentry AB, Acosta RD, Greenwald BD, Riddle M. Endoscopic ultrasound does not accurately stage early adenocarcinoma or high-grade dysplasia of the esophagus. Clin Gastroenterol Hepatol. 2010;8:1037-41.

15. Choi J, Kim SG, Kim JS, Jung HC, Song IS. Comparison of endoscopic ultrasonography (EUS), positron emission tomography (PET), and computed tomography (CT) in the preoperative locoregional staging of resectable esophageal cancer. Surg Endosc. 2010;24:1380-6.

16. McDonough PB, Jones DR, Shen KR, Northup PG, Galysh RL, Hernandez A, et al. Does FDG-PET add information to EUS and CT in the initial management of esophageal cancer? A prospective single center study. Am J Gastroenterol. 2008;103:570-4.

17. Luketich JD, Meehan M, Nguyen NT, Christie N, Weigel T, Yousem S, et al. Minimally invasive surgical staging for esophageal cancer. Surg Endosc. 2000;14:700-2.

18. Abate E, DeMeester SR, Zehetner J, Oezcelik A, Ayazi S, Costales J, et al. Recurrence after esophagectomy for adenocarcinoma: defining optimal follow-up intervals and testing. J Am Coll Surg. 2010;210:428-35. 
19. Meguid RA, Hooker CM, Taylor JT, Kleinberg LR, Cattaneo SM II, Sussman MS, et al. Recurrence after neoadjuvant chemoradiation and surgery for esophageal cancer: does the pattern of recurrence differ for patients with complete response and those with partial or no response? J Thorac Cardiovasc Surg. 2009;138:1309-17.

20. Ando N, Iizuka T, Ide H, Ishida K, Shinoda M, Nishimaki T, et al. Surgery plus chemotherapy compared with surgery alone for localized squamous cell carcinoma of the thoracic esophagus: a Japan Clinical Oncology Group StudyJCOG9204. J Clin Oncol. 2003;21:4592-6.

21. Spizzo G, Ofner D, de Vries A, Lukas P, Steger G, Pluschnig U, et al. Preoperative chemotherapy with cisplatin and docetaxel followed by surgery and clip-oriented postoperative chemoradiation in patients with localized gastric or gastroesophageal junction adenocarcinoma: results from a phase II feasibility study. Ann Surg Oncol. 2011;18:677-83.

22. Levy RM, Luketich JD. Minimally invasive intrathoracic esophagogastric anastomosis. Semin Thorac Cardiovasc Surg. 2010;22:256-8.

23. Pennathur A, Awais O, Luketich JD. Technique of minimally invasive Ivor Lewis esophagectomy. Ann Thorac Surg. 2010;89:S2159-62.

24. Luketich JD, Alvelo-Rivera M, Buenaventura PO, Christie NA, McCaughan JS, Litle VR, et al. Minimally invasive esophagectomy: outcomes in 222 patients. Ann Surg. 2003;238:486-94; discussion 94-5.

25. Luketich JD, Pennathur A, Awais O, Levy RM, Keeley S, Shende M, et al. Outcomes after minimally invasive esophagectomy: review of over 1000 patients. Ann Surg. 2012;256:95-103.

26. Page DL, Fleming ID, Fritz A. AJCC Cancer Staging Manual. 6th edition. Philadelphia, PA: Lippincott-Raven; 2002.

27. Charlson ME, Pompei P, Ales KL, MacKenzie CR. A new method of classifying prognostic comorbidity in longitudinal studies: development and validation. J Chronic Dis. 1987;40:373-83.

28. StataCorp. Stata Statistical Software: Release 13. College Station, TX: StataCorp LP; 2013.

29. Edge SE, Byrd DR, Compton CC, Fritz AG, Greene FL, Trotti A. AJCC Cancer Staging Manual. 7th edition. New York, NY: Springer; 2009.

30. Welsh J, Settle SH, Amini A, Xiao L, Suzuki A, Hayashi Y, et al. Failure patterns in patients with esophageal cancer treated with definitive chemoradiation. Cancer. 2012;118:2632-40.

31. McKenzie S, Mailey B, Artinyan A, Metchikian M, Shibata S, Kernstine K, et al. Improved outcomes in the management of esophageal cancer with the addition of surgical resection to chemoradiation therapy. Ann Surg Oncol. 2011;18:551-8.

32. Tougeron D, Scotte M, Hamidou H, Di Fiore F, Paillot B, Michot F, et al. Definitive chemoradiotherapy in patients with esophageal adenocarcinoma: an alternative to surgery? J Surg Oncol. 2012;105:761-6.

33. van Hagen P, Hulshof MC, van Lanschot JJ, Steyerberg EW, van Berge Henegouwen MI, Wijnhoven BP, et al. Preoperative chemoradiotherapy for esophageal or junctional cancer. N Engl J Med. 2012;366:2074-84.

34. Hammoud ZT, Kesler KA, Ferguson MK, Battafarrano RJ, Bhogaraju A, Hanna N, et al. Survival outcomes of resected patients who demonstrate a path- ologic complete response after neoadjuvant chemoradiation therapy for locally advanced esophageal cancer. Dis Esophagus. 2006;19:69-72.

35. Gaca JG, Petersen RP, Peterson BL, Harpole DH Jr, D’Amico TA, Pappas TN, et al. Pathologic nodal status predicts disease-free survival after neoadjuvant chemoradiation for gastroesophageal junction carcinoma. Ann Surg Oncol. 2006;13: 340-6.

36. Agarwal B, Swisher SG, Ajani J, Kelly K, Komaki RR, Abu-Hamda E, et al. Differential response to preoperative chemoradiation and surgery in esophageal adenocarcinomas based on presence of Barrett's esophagus and symptomatic gastroesophageal reflux. Ann Thorac Surg. 2005;79:1716-23.

37. Urschel JD, Vasan H. A meta-analysis of randomized controlled trials that compared neoadjuvant chemoradiation and surgery to surgery alone for resectable esophageal cancer. Am J Surg. 2003;185:538-43.

38. Urschel JD, Vasan H, Blewett CJ. A meta-analysis of randomized controlled trials that compared neoadjuvant chemotherapy and surgery to surgery alone for resectable esophageal cancer. Am J Surg. 2002;183:274-9.

39. Bang YJ, Van Cutsem E, Feyereislova A, Chung HC, Shen L, Sawaki A, et al. Trastuzumab in combination with chemotherapy versus chemotherapy alone for treatment of HER2-positive advanced gastric or gastro-oesophageal junction cancer (ToGA): a phase 3, open-label, randomised controlled trial. Lancet. 2010; 376:687-97.

40. Donahue JM, Nichols FC, Li Z, Schomas DA, Allen MS, Cassivi SD, et al. Complete pathologic response after neoadjuvant chemoradiotherapy for esophagea cancer is associated with enhanced survival. Ann Thorac Surg. 2009;87:392-8; discussion 8-9.

41. Schneider PM, Baldus SE, Metzger R, Kocher M, Bongartz R, Bollschweiler E, et al. Histomorphologic tumor regression and lymph node metastases determine prognosis following neoadjuvant radiochemotherapy for esophageal cancer: implications for response classification. Ann Surg. 2005;242:684-92.

42. McHorney CA, Ware JE Jr. Construction and validation of an alternate form general mental health scale for the Medical Outcomes Study Short-Form 36-Item Health Survey. Med Care. 1995;33:15-28.

43. Biere SS, Maas KW, Bonavina L, Garcia JR, van Berge Henegouwen MI, Rosman C, et al. Traditional invasive vs. minimally invasive esophagectomy: a multi-center, randomized trial (TIME-trial). BMC Surg. 2011;11:2.

44. Parameswaran R, Titcomb DR, Blencowe NS, Berrisford RG, Wajed SA, Streets CG, et al. Assessment and comparison of recovery after open and minimally invasive esophagectomy for cancer: an exploratory study in two centers. Ann Surg Oncol. 2013;20:1970-7.

45. Little RJ, Rubin DB. The central role of the propensity score in observational studies for causal effects. Biometrika. 1983;70:41-55.

Key words: neoadjuvant therapy, esophagectomy, propensity score, surgical procedures, minimally invasive, mortality 